\title{
Two-Dimensional and Axisymmetric Unsteady Flows due to Normally Expanding or Contracting Parallel Plates
}

\author{
Saeed Dinarvand ${ }^{\mathbf{1}}$ and Abed Moradi ${ }^{2}$ \\ ${ }^{1}$ Young Researchers Club, Islamic Azad University, Central Tehran Branch, Tehran, Iran \\ ${ }^{2}$ Mechanical Engineering Department, Islamic Azad University, Central Tehran Branch, Tehran, Iran \\ Correspondence should be addressed to Saeed Dinarvand, saeed_dinarvand@yahoo.com
}

Received 17 December 2011; Accepted 22 February 2012

Academic Editor: Md. Sazzad Chowdhury

Copyright (C) 2012 S. Dinarvand and A. Moradi. This is an open access article distributed under the Creative Commons Attribution License, which permits unrestricted use, distribution, and reproduction in any medium, provided the original work is properly cited.

\begin{abstract}
The flow of a viscous incompressible fluid between two parallel plates due to the normal motion of the plates for two cases, the two-dimensional flow case and the axisymmetric flow case, is investigated. The governing nonlinear equations and their associated boundary conditions are transformed into a highly non-linear ordinary differential equation. The series solution of the problem is obtained by utilizing the homotopy perturbation method (HPM). Graphical results are presented to investigate the influence of the squeeze number on the velocity, skin friction, and pressure gradient. The validity of our solutions is verified by the numerical results obtained by shooting method, coupled with Runge-Kutta scheme.
\end{abstract}

\section{Introduction}

Most of the scientific problems and phenomena are modeled by nonlinear ordinary or partial differential equations. In recent years, many powerful methods have been developed to construct explicit analytical solution of nonlinear differential equations. Among them, two analytical methods have drawn special attention, namely, the homotopy perturbation method (HPM) [1, 2] and homotopy analysis method (HAM) [3-6]. The essential idea in these methods is to introduce a homotopy parameter, say $p$, which takes the value from 0 to 1 . For $p=0$, the system of equations takes a simplified form which readily admits a particularly simple solution. When $p$ is gradually increased to 1 , the system goes through a sequence of deformations, the solution of each of which is close to that at the previous stage of deformation. Eventually at $p=1$, the system takes the original forms of equation, and the final stage of deformation gives the desired solution.

We know that all perturbation methods require small parameter in nonlinear equation, and the approximate solutions of equation containing this parameter are expressed as series 
expansions in the small parameter. Selection of small parameter requires a special skill. A proper choice of small parameter gives acceptable results, while an improper choice may result in incorrect solutions. The homotopy perturbation method, which is a coupling of the traditional perturbation method and homotopy in topology, does not require a small parameter in equation modeling phenomena. In recent years, the HPM has been successfully employed to solve many types of linear and nonlinear problems such as the quadratic Riccati differential equation [7], the axisymmetric flow over a stretching sheet [8], the fractional Fokker-Planck equations [9], the magnetohydrodynamic flow over a nonlinear stretching sheet [10], the thin film flow of a fourth grade fluid down a vertical cylinder [11], the fractional diffusion equation with absorbent term and external force [12], Burgers equation with finite transport memory [13], the system of Fredholm integral equations [14], the generalized Burger and Burger-Fisher equations [15], the wave and nonlinear diffusion equations [16], the flow through slowly expanding or contracting porous walls [17], the torsional flow of third-grade fluid [18], Emden-Fowler equations [19], and the long porous slider [20]. All of these successful applications verified the validity, effectiveness, and flexibility of the HPM.

The problem of unsteady squeezing of a viscous incompressible fluid between two parallel plates in motion normal to their own surfaces independent of each other and arbitrary with respect to time is a fundamental type of unsteady flow which is met frequently in many hydrodynamical machines and apparatus. Some practical examples of squeezing flow include polymer processing, compression, and injection molding. In addition, the lubrication system can also be modeled by squeezing flows. Stefan [21] published a classical paper on squeezing flow by using lubrication approximation. In 1886, Reynolds [22] obtained a solution for elliptic plates, and Archibald [23] studied this problem for rectangular plates. The theoretical and experimental studies of squeezing flows have been conducted by many researchers [24-35]. Earlier studies of squeezing flow are based on Reynolds equation. The inadequacy of Reynolds equation in the analysis of porous thrust bearings and squeeze films involving high velocity has been demonstrated by Jackson [34], Ishizawa [35], and others. The general study of the problem with full Navier-Stokes equations involves extensive numerical study requiring more computer time and larger memory. However, many of the important features of this problem can be grasped by prescribing the relative velocity of the plates suitably. If the relative normal velocity is proportional to $(1-\alpha t)^{1 / 2}$, where $t$ is the time and $\alpha$ a constant of dimension $\left[T^{-1}\right]$ which characterizes unsteadiness, then the unsteady Navier-Stokes equations admit similarity solution.

With the above discussion in mind, the purpose of the present paper is to examine analytically the problem of unsteady flows due to normally expanding or contracting parallel plates. The governing equations here are highly nonlinear coupled differential equations, which are solved by using the homotopy perturbation method. In this way, the paper has been organized as follows. In Section 2, the problem statement and mathematical formulation are presented. In Section 3, we extend the application of the HPM to construct the approximate solution for the governing equations. Section 4 contains the results and discussion. The conclusions are summarized in Section 5 .

\section{Flow Development and Mathematical Formulation}

Let the position of the two plates be at $z= \pm \ell(1-\alpha t)^{1 / 2}$, where $\ell$ is the position at time $t=0$ as shown in Figure 1. We assume that the length 1 (in the two-dimensional case) or the 


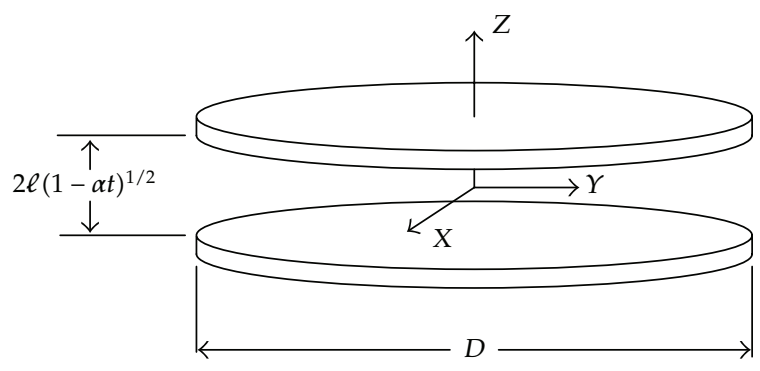

Figure 1: Schematic diagram of the problem.

diameter $D$ (in the axisymmetric case) are much larger than the gap width $2 z$ at any time such that the end effects can be neglected. When $\alpha$ is positive, the two plates are squeezed until they touch at $t=1 / \alpha$. When $\alpha$ is negative, the two plates are separated. Let $u, v$, and $w$ be the velocity components in the $x, y$, and $z$ directions, respectively. For two-dimensional flow, Wang introduced the following transforms [36]:

$$
\begin{gathered}
u=\frac{\alpha x}{[2(1-\alpha t)]} f^{\prime}(\eta), \\
w=\frac{-\alpha \ell}{\left[2(1-\alpha t)^{1 / 2}\right]} f(\eta),
\end{gathered}
$$

where

$$
\eta=\frac{z}{\left[\ell(1-\alpha t)^{1 / 2}\right]} .
$$

Substituting (2.1) into the unsteady two-dimensional Navier-Stokes equations yields nonlinear ordinary differential equation in form:

$$
f^{\prime \prime \prime \prime}+S\left\{-\eta f^{\prime \prime \prime}-3 f^{\prime \prime}-f^{\prime} f^{\prime \prime}+f f^{\prime \prime \prime}\right\}=0,
$$

where $S=\alpha \ell^{2} / 2 v$ (squeeze number) is the nondimensional parameter. The flow is characterized by this parameter. The boundary conditions are such that on the plates the lateral velocities are zero and the normal velocity is equal to the velocity of the plate, that is,

$$
\begin{array}{ll}
f(0)=0, & f^{\prime \prime}(0)=0, \\
f(1)=1, & f^{\prime}(1)=0 .
\end{array}
$$


Similarly, the Wang's transforms [36] for axisymmetric flow are

$$
\begin{gathered}
u=\frac{\alpha x}{[4(1-\alpha t)]} f^{\prime}(\eta), \\
v=\frac{\alpha y}{[4(1-\alpha t)]} f^{\prime}(\eta), \\
w=\frac{-\alpha \ell}{\left[2(1-\alpha t)^{1 / 2}\right]} f(\eta) .
\end{gathered}
$$

Using transforms (2.5), unsteady axisymmetric Navier-Stokes equations reduce to

$$
f^{\prime \prime \prime \prime}+S\left\{-\eta f^{\prime \prime \prime}-3 f^{\prime \prime}+f f^{\prime \prime \prime}\right\}=0,
$$

subject to the boundary conditions (2.4).

Consequently, we should solve the nonlinear ordinary differential equation

$$
f^{\prime \prime \prime \prime}+S\left\{-\eta f^{\prime \prime \prime}-3 f^{\prime \prime}-\beta f^{\prime} f^{\prime \prime}+f f^{\prime \prime \prime}\right\}=0,
$$

where

$$
\beta= \begin{cases}0, & \text { Axisymmetric } \\ 1, & \text { Two-dimensional }\end{cases}
$$

and subject to boundary conditions (2.4).

\section{Solution by Homotopy Perturbation Method}

\subsection{Basic Idea}

Now, for convenience, consider the following general nonlinear differential equation

$$
A(u)-f(r)=0, \quad r \in \Omega,
$$

with boundary conditions

$$
B\left(u, \frac{\partial u}{\partial n}\right)=0, \quad r \in \Gamma
$$

where $A$ is a general differential operator, $B$ is a boundary operator, $f(r)$ is a known analytic function, and $\Gamma$ is the boundary of the domain $\Omega$.

The operator $A$ can, generally speaking, be divided into two parts $L$ and $N$, where $L$ is linear and $N$ is nonlinear; therefore (3.1) can be written as

$$
L(u)+N(u)-f(r)=0 .
$$


By using homotopy technique, one can construct a homotopy $v(r, p): \Omega \times[0,1] \rightarrow \mathcal{R}$ which satisfies homotopy equation:

$$
H(v, p)=(1-p)\left[L(v)-L\left(u_{0}\right)\right]+p[A(v)-f(r)]=0,
$$

or

$$
H(v, p)=L(v)-L\left(u_{0}\right)+p L\left(u_{0}\right)+p[N(v)-f(r)]=0,
$$

where $P \in[0,1]$ is an embedding parameter and $u_{0}$ is the initial approximation of (3.1) which satisfies the boundary conditions. Clearly, we have

$$
\begin{gathered}
H(v, 0)=L(v)-L\left(u_{0}\right)=0, \\
H(v, 1)=A(v)-f(r)=0 .
\end{gathered}
$$

The changing process of $p$ from zero to unity is just that of $v(r, p)$ changing from $u_{0}(r)$ to $u(r)$. This is called deformation, and, also, $L(v)-L\left(u_{0}\right)$ and $A(v)-f(r)$ are called homotopic in topology. If the embedding parameter $p(0 \leq p \leq 1)$ is considered as a small parameter, applying the classical perturbation technique, we can naturally assume that the solution of (3.4) and (3.5) can be given as a power series in $p$, that is,

$$
v=v_{0}+p v_{1}+p^{2} v_{2}+\cdots
$$

and setting $p=1$ results in the approximate solution of (3.8) as

$$
u=\lim _{p \rightarrow 1} v=v_{0}+v_{1}+v_{2}+\cdots .
$$

The convergence of series (3.8) has been proved by He in his paper [37]. It is worth to note that the major advantage of He's homotopy perturbation method is that the perturbation equation can be freely constructed in many ways (therefore is problem dependent) by homotopy in topology and the initial approximation can also be freely selected. Moreover, the construction of the homotopy for the perturb problem plays very important role for obtaining desired accuracy.

\subsection{Guidelines for Choosing Homotopy Equation}

In a homotopy equation, what we are mainly concerned about are the auxiliary linear operator $L$ and the initial approximation $u_{0}$. Once one chooses these parts, the homotopy equation is completely determined, because the remaining part is actually the original equation (see (3.12)), and we have less freedom to change it. Here we discuss some general rules that should be noted in choosing $L$ and $u_{0}$.

\subsubsection{Discussion on Auxiliary Linear Operator L}

According to the steps of the homotopy perturbation method, $L$ should be as follows. 
(i) Easy to Handle

We mean that it must be chosen in such a way that one has no difficulty in subsequently solving systems of resulting equations [38]. It should be noted that this condition does not restrict $L$ to be linear. In scarce cases, as was done by He in [37] to solve the Lighthill equation, a nonlinear choice of $L$ may be more suitable. But, it is strongly recommended for beginners to take a linear operator as $L$.

\section{(ii) Closely Related to the Original Equation}

Strictly speaking, in constructing $L$, it is better to use some part of the original equation [39]. We can see the effectiveness of this view in [40] where Chowdhury and Hashim have gained very good results with technically choosing the $L$ part.

\subsubsection{Discussion on Initial Approximation $u_{0}$}

There is no unique universal technique for choosing the initial approximation in iterative methods, but from previous works done on HPM [41, 42] and our own experiences, we can conclude the following facts.

\section{(i) It Should Be Obtained from the Original Equation}

For example, it can be chosen to be the solution to some part of the original equation, or it can be chosen from initial/boundary conditions.

\section{(ii) It Should Reduce Complexity of the Resulting Equations}

Although this condition only can be checked after solving some of the first few equations of the resulting system, these are the criteria that have been used by many authors when they encountered different choices as an initial approximation.

\subsection{Application for Unsteady Flows due to Normally Expanding or Contracting Parallel Plates}

To investigate the explicit and totally analytic solutions of present problem by using HPM, we first define homotopy $v(\eta, p): \Omega \times[0,1] \rightarrow \mathcal{R}$ for (2.7) which satisfies

$$
(1-p)\left[L(v)-L\left(f_{0}\right)\right]+p\left[v^{I V}+S\left(-\eta v^{\prime \prime \prime}-3 v^{\prime \prime}-\beta v^{\prime} v^{\prime \prime}+v v^{\prime \prime \prime}\right)\right]=0,
$$

where $L$ is linear operators as follows:

$$
L(v)=\frac{d^{4} v}{d \eta^{4}}
$$


We choose

$$
f_{0}(\eta)=\frac{1}{2}\left(3 \eta-\eta^{3}\right)
$$

as initial approximation of $f(\eta)$, which satisfy the boundary conditions (2.4). Assume that the solution of equation (3.9) has the form

$$
v(\eta)=v_{0}(\eta)+p v_{1}(\eta)+p^{2} v_{2}(\eta)+\cdots
$$

where $v_{i}(\eta), i=1,2,3, \ldots$ are functions yet to be determined. Substituting (3.12) into (3.9) and equating the terms with identical powers of $p$, we have

$$
\begin{aligned}
& p^{0} \Longrightarrow v_{0}^{\prime \prime \prime \prime}-f_{0}^{\prime \prime \prime \prime}=0 \text {, } \\
& v_{0}(0)=0, \quad v_{0}^{\prime \prime}(0)=0, \\
& v_{0}(1)=1, \quad v_{0}^{\prime}(1)=0, \\
& p^{1} \Longrightarrow v_{1}^{\prime \prime \prime \prime}+f_{0}^{\prime \prime \prime \prime}+S\left\{-\eta v_{0}^{\prime \prime \prime}-3 v_{0}^{\prime \prime}-\beta v_{0}^{\prime} v_{0}^{\prime \prime}+v_{0} v_{0}^{\prime \prime \prime}\right\}=0 \text {, } \\
& v_{1}(0)=0, \quad v_{1}^{\prime \prime}(0)=0, \\
& v_{1}(1)=0, \quad v_{1}^{\prime}(1)=0, \\
& p^{2} \Longrightarrow v_{2}^{\prime \prime \prime \prime}+S\left\{-\eta v_{1}^{\prime \prime \prime}-3 v_{1}^{\prime \prime}-\beta\left(v_{1}^{\prime} v_{0}^{\prime \prime}+v_{0}^{\prime} v_{1}^{\prime \prime}\right)+\left(v_{1} v_{0}^{\prime \prime \prime}+v_{0} v_{1}^{\prime \prime \prime}\right)\right\}=0 \text {, } \\
& v_{2}(0)=0, \quad v_{2}^{\prime \prime}(0)=0, \\
& v_{2}(1)=0, \quad v_{2}^{\prime}(1)=0, \\
& p^{3} \Longrightarrow v_{3}^{\prime \prime \prime \prime}+S\left\{-\eta v_{2}^{\prime \prime \prime}-3 v_{2}^{\prime \prime}-\beta\left(v_{2}^{\prime} v_{0}^{\prime \prime}+v_{1}^{\prime} v_{1}^{\prime \prime}+v_{0}^{\prime} v_{2}^{\prime \prime}\right)+\left(v_{2} v_{0}^{\prime \prime \prime}+v_{1} v_{1}^{\prime \prime \prime}+v_{0} v_{2}^{\prime \prime \prime}\right)\right\}=0, \\
& v_{3}(0)=0, \quad v_{3}^{\prime \prime}(0)=0 \text {, } \\
& v_{3}(1)=0, \quad v_{3}^{\prime}(1)=0, \\
& p^{4} \Longrightarrow v_{4}^{\prime \prime \prime \prime}+S\left\{-\eta v_{3}^{\prime \prime \prime}-3 v_{3}^{\prime \prime}-\beta\left(v_{3}^{\prime} v_{0}^{\prime \prime}+v_{2}^{\prime} v_{1}^{\prime \prime}+v_{1}^{\prime} v_{2}^{\prime \prime}+v_{0}^{\prime} v_{3}^{\prime \prime}\right)+\left(v_{3} v_{0}^{\prime \prime \prime}+v_{2} v_{1}^{\prime \prime \prime}+v_{1} v_{2}^{\prime \prime \prime}+v_{0} v_{3}^{\prime \prime \prime}\right)\right\}=0 \text {, } \\
& v_{4}(0)=0, \quad v_{4}^{\prime \prime}(0)=0, \\
& v_{4}(1)=0, \quad v_{4}^{\prime}(1)=0, \\
& p^{5} \Longrightarrow v_{5}^{\prime \prime \prime \prime}+S\left\{-\eta v_{4}^{\prime \prime \prime}-3 v_{4}^{\prime \prime}-\beta\left(v_{4}^{\prime} v_{0}^{\prime \prime}+v_{3}^{\prime} v_{1}^{\prime \prime}+v_{2}^{\prime} v_{2}^{\prime \prime}+v_{1}^{\prime} v_{3}^{\prime \prime}+v_{0}^{\prime} v_{4}^{\prime \prime}\right)\right. \\
& \left.+\left(v_{4} v_{0}^{\prime \prime \prime}+v_{3} v_{1}^{\prime \prime \prime}+v_{2} v_{2}^{\prime \prime \prime}+v_{1} v_{3}^{\prime \prime \prime}+v_{0} v_{4}^{\prime \prime \prime}\right)\right\}=0, \\
& v_{5}(0)=0, \quad v_{5}^{\prime \prime}(0)=0 \text {, } \\
& v_{5}(1)=0, \quad v_{5}^{\prime}(1)=0, \\
& p^{6} \Longrightarrow v_{6}^{\prime \prime \prime \prime}+S\left\{-\eta v_{5}^{\prime \prime \prime}-3 v_{5}^{\prime \prime}-\beta\left(v_{5}^{\prime} v_{0}^{\prime \prime}+v_{4}^{\prime} v_{1}^{\prime \prime}+v_{3}^{\prime} v_{2}^{\prime \prime}+v_{2}^{\prime} v_{3}^{\prime \prime}+v_{1}^{\prime} v_{4}^{\prime \prime}+v_{0}^{\prime} v_{5}^{\prime \prime}\right)\right. \\
& \left.+\left(v_{5} v_{0}^{\prime \prime \prime}+v_{4} v_{1}^{\prime \prime \prime}+v_{3} v_{2}^{\prime \prime \prime}+v_{2} v_{3}^{\prime \prime \prime}+v_{1} v_{4}^{\prime \prime \prime}+v_{0} v_{5}^{\prime \prime \prime}\right)\right\}=0, \\
& v_{6}(0)=0, \quad v_{6}^{\prime \prime}(0)=0 \text {, } \\
& v_{6}(1)=0, \quad v_{6}^{\prime}(1)=0 \text {. }
\end{aligned}
$$


Table 1: The analytic results of $f(\eta)$ at different terms of approximation compared with the numerical results (RK4) for the axisymmetric case.

\begin{tabular}{cccccc}
\hline$S$ & $\eta$ & 3 Terms & 5 Terms & 7 Terms & Numerical (RK4) \\
\hline \multirow{4}{*}{-1.5} & 0.2 & 0.319474 & 0.319526 & 0.319526 & 0.319526 \\
& 0.4 & 0.603652 & 0.603825 & 0.603830 & 0.603830 \\
& 0.6 & 0.822574 & 0.822863 & 0.822875 & 0.822876 \\
& 0.8 & 0.956580 & 0.956789 & 0.956800 & 0.956801 \\
\hline \multirow{4}{*}{0.5} & 0.2 & 0.302545 & 0.302582 & 0.302582 & 0.302582 \\
& 0.4 & 0.578028 & 0.578082 & 0.578082 & 0.578082 \\
& 0.6 & 0.800737 & 0.800780 & 0.800780 & 0.800780 \\
& 0.8 & 0.947686 & 0.947702 & 0.947702 & 0.947702 \\
\hline \multirow{3}{*}{0.5} & 0.2 & 0.290353 & 0.290322 & 0.290322 & 0.290322 \\
& 0.4 & 0.559299 & 0.559253 & 0.559252 & 0.559252 \\
& 0.6 & 0.784341 & 0.784304 & 0.784303 & 0.784303 \\
& 0.8 & 0.940717 & 0.940704 & 0.940703 & 0.940703 \\
\hline \multirow{4}{*}{1.5} & 0.2 & 0.281032 & 0.281010 & 0.281010 & 0.281010 \\
& 0.4 & 0.544851 & 0.544780 & 0.544779 & 0.544779 \\
& 0.6 & 0.771493 & 0.771374 & 0.771371 & 0.771371 \\
& 0.8 & 0.935127 & 0.935038 & 0.935036 & 0.935036 \\
\hline
\end{tabular}

Using the Mathematica package, the solutions of system (3.13) may be written as follows:

$$
\begin{aligned}
& v_{0}(\eta)=\frac{1}{2}\left(3 \eta-\eta^{3}\right) \\
& v_{1}(\eta)=\frac{S}{560}\left\{(37+15 \beta) \eta-(73+33 \beta) \eta^{3}+(35+21 \beta) \eta^{5}-(-1+3 \beta) \eta^{7}\right\}, \\
& v_{2}(\eta)=\frac{S}{15523200}\left\{\left(\begin{array}{c}
1025640+415800 \beta-1025640 \\
-415800 \beta-153060 S-126789 S \beta \\
-25875 S \beta^{2}
\end{array}\right) \eta\right. \\
& -\left(\begin{array}{c}
2023560+914760 \beta-2023560 \\
-914760 \beta-349010 S-325392 S \beta \\
-73998 S \beta^{2}
\end{array}\right) \eta^{3} \\
& +\left(\begin{array}{c}
970200+582120 \beta-970200 \\
-582120 \beta-227304 S-281358 S \beta \\
-79002 S \beta^{2}
\end{array}\right) \eta^{5} \\
& -\left(\begin{array}{c}
-27720+83160 \beta+27720 \\
-83160 \beta-20196 S-92268 S \beta \\
-40392 S \beta^{2}
\end{array}\right) \eta^{7} \\
& \left.+\left(\begin{array}{c}
-10780 S \\
-8085 S \beta \\
-10395 S \beta^{2}
\end{array}\right) \eta^{9}-\left(\begin{array}{c}
-378 S \\
+1428 S \beta \\
-882 S \beta^{2}
\end{array}\right) \eta^{11}\right\},
\end{aligned}
$$


Table 2: The analytic results of $f(\eta)$ at different terms of approximation compared with the numerical results (RK4) for the two-dimensional case.

\begin{tabular}{cccccc}
\hline$S$ & $\eta$ & 3 Terms & 5 Terms & 7 Terms & Numerical (RK4) \\
\hline \multirow{4}{*}{-1.5} & 0.2 & 0.332883 & 0.333591 & 0.333617 & 0.333618 \\
& 0.4 & 0.623190 & 0.624315 & 0.624358 & 0.624358 \\
& 0.6 & 0.838219 & 0.839284 & 0.839324 & 0.839325 \\
& 0.8 & 0.962441 & 0.962961 & 0.962983 & 0.962984 \\
\hline \multirow{4}{*}{0.5} & 0.2 & 0.305436 & 0.305543 & 0.305545 & 0.305545 \\
& 0.4 & 0.582314 & 0.582468 & 0.582470 & 0.582470 \\
& 0.6 & 0.804271 & 0.804390 & 0.804392 & 0.804392 \\
& 0.8 & 0.949065 & 0.949107 & 0.949108 & 0.949108 \\
\hline \multirow{4}{*}{0.5} & 0.2 & 0.288347 & 0.288261 & 0.288260 & 0.288260 \\
& 0.4 & 0.556268 & 0.556145 & 0.556143 & 0.556143 \\
& 0.6 & 0.781768 & 0.781670 & 0.781671 & 0.781671 \\
& 0.8 & 0.939674 & 0.939641 & 0.939640 & 0.939640 \\
\hline \multirow{4}{*}{1.5} & 0.2 & 0.276526 & 0.276433 & 0.276432 & 0.276432 \\
& 0.4 & 0.537929 & 0.537754 & 0.537752 & 0.537752 \\
& 0.6 & 0.765463 & 0.765252 & 0.765249 & 0.765249 \\
\hline
\end{tabular}

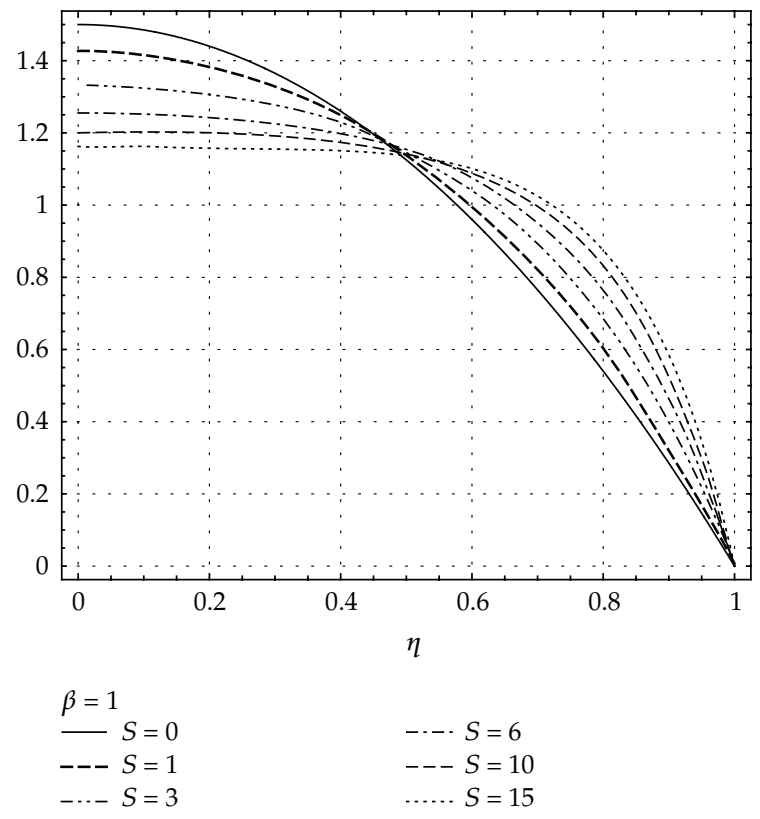

Figure 2: The influence of positive $S$ on $f^{\prime}(\eta)$ for the two-dimensional case.

Other terms are too long for presentation. According to the HPM, we can conclude that

$$
f(\eta)=\lim _{p \rightarrow 1} v(\eta)=v_{0}(\eta)+v_{1}(\eta)+v_{2}(\eta)+v_{3}(\eta)+v_{4}(\eta)+v_{5}(\eta)+v_{6}(\eta)
$$




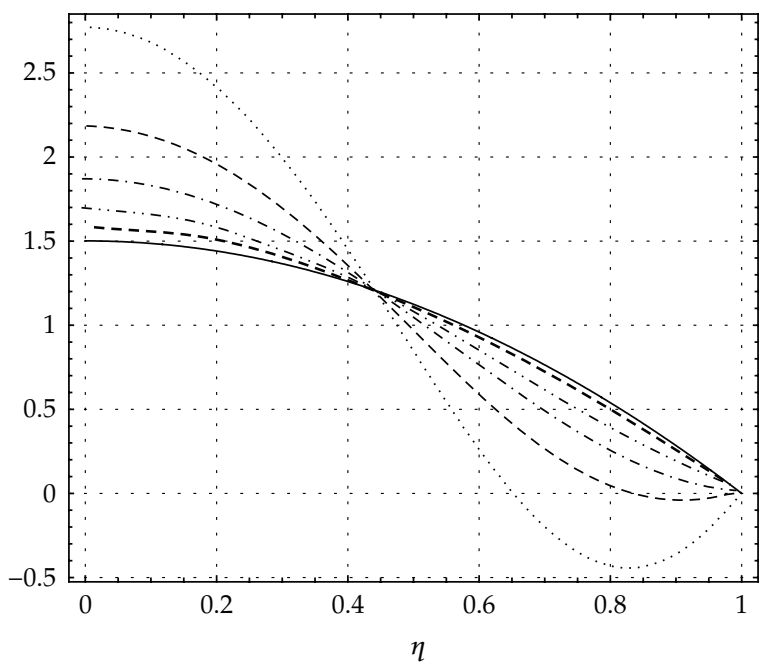

$$
\begin{aligned}
& \beta=0 \\
& -S=-0.1 \\
& \text {--- } S=-1 \\
& \ldots-S=-2 \\
& \text {-.-. } S=-3 \\
& ---S=-4 \\
& \text { …. } S=-5
\end{aligned}
$$

Figure 3: The influence of negative $S$ on $f^{\prime}(\eta)$ for the axisymmetric case.

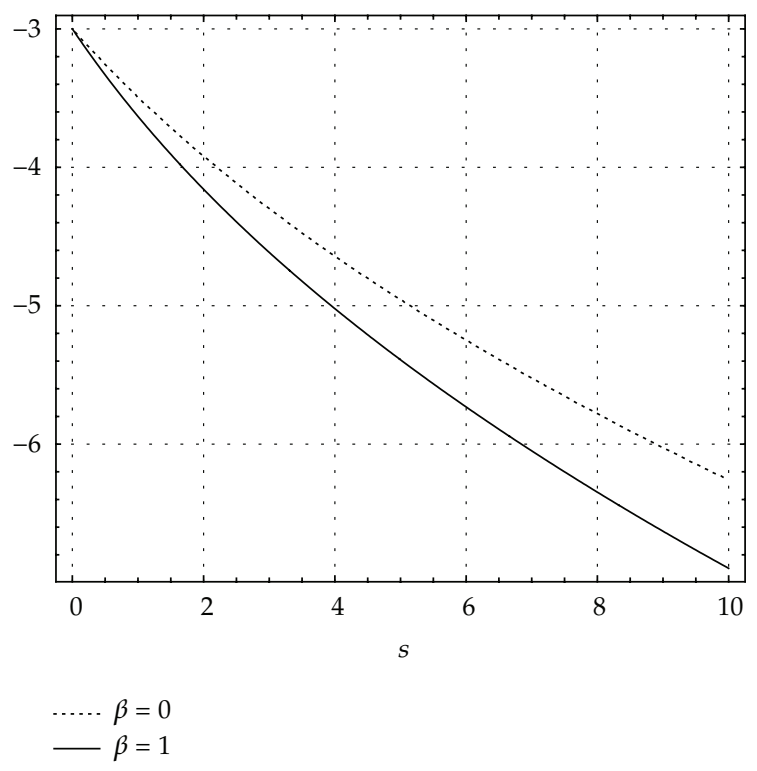

Figure 4: The skin friction $\left(f^{\prime \prime}(1)\right)$ for the axisymmetric and two-dimensional cases.

\section{Results and Discussion}

The fourth-order ordinary differential equation (3.2), with the boundary conditions (3.3), is solved numerically using shooting method, coupled with Runge-Kutta scheme. Our main concern is the various values of $f(\eta)$ and $f^{\prime}(\eta)$. These quantities describe the flow behaviour. 


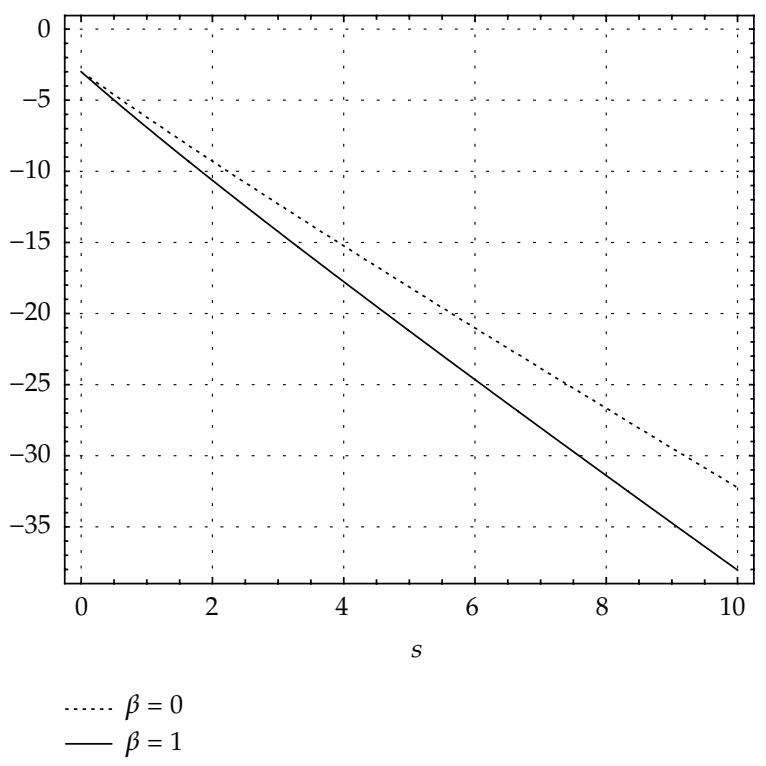

Figure 5: The pressure gradient $\left(f^{\prime \prime \prime}(1)\right)$ for the axisymmetric and two-dimensional cases.

For several values of $S$, the function $f(\eta)$ obtained by the different order of approximation for the axisymmetric and two-dimensional cases are compared with the numerical results in Tables 1 and 2, respectively. We can see a very good agreement between the purely analytic results of the HAM and numerical results. The variation of $f^{\prime}(\eta)$ with the change in the positive values of $S$ for the two-dimensional case is plotted in Figure 2. Figure 3 shows the influence of negative $S$ on $f^{\prime}(\eta)$ for the axisymmetric case. Note that for the large negative values of $S$, the results of similarity analysis are not reliable. $f^{\prime \prime}(1)$ gives skin friction, and $f^{\prime \prime \prime}(1)$ represents pressure gradient. $f^{\prime \prime}(1)$ and $f^{\prime \prime \prime}(1)$ as functions of $S$ are illustrated in Figures 4 and 5 , respectively.

\section{Conclusions}

In this paper, the unsteady axisymmetric and two-dimensional squeezing flows between two parallel plates are studied using the homotopy perturbation method (HPM). Graphical results and tables are presented to investigate the influence of the squeeze number on the velocity, skin friction, and pressure gradient. Here, the results are compared with the numerical solution obtained using shooting method, coupled with Runge-Kutta scheme. The obtained solutions, in comparison with the numerical solutions, demonstrate remarkable accuracy. This method provides an analytical approximate solution without any assumption of linearization. This character is very important for equations with strong nonlinearities which could be extremely sensitive to small changes in parameters. In this regard the homotopy perturbation method is found to be a very useful analytic technique to get highly accurate and purely analytic solution to such kind of nonlinear problems.

\section{References}

[1] J.-H. He, "A coupling method of a homotopy technique and a perturbation technique for non-linear problems," International Journal of Non-Linear Mechanics, vol. 35, no. 1, pp. 37-43, 2000. 
[2] J.-H. He, "Homotopy perturbation method for solving boundary value problems," Physics Letters A, vol. 350 , no. 1-2, pp. 87-88, 2006.

[3] Liao, The proposed homotopy analysis technique for the solution of non-linear problems, Ph.D. thesis, Shanghai Jiao Tong University, 1992.

[4] S. Dinarvand, "A reliable treatment of the homotopy analysis method for viscous flow over a nonlinearly stretching sheet in presence of a chemical reaction and under influence of a magnetic field," Central European Journal of Physics, vol. 7, no. 1, pp. 114-122, 2009.

[5] S. Dinarvand, "On explicit, purely analytic solutions of off-centered stagnation flow towards a rotating disc by means of HAM," Nonlinear Analysis. Real World Applications, vol. 11, no. 5, pp. 3389 3398, 2010.

[6] S. Dinarvand, "The laminar free-convection boundary-layer flow about a heated and rotating downpointing vertical cone in the presence of a transverse magnetic field," International Journal for Numerical Methods in Fluids, vol. 67, no. 12, pp. 2141-2156, 2011.

[7] S. Abbasbandy, "Iterated He's homotopy perturbation method for quadratic Riccati differential equation," Applied Mathematics and Computation, vol. 175, no. 1, pp. 581-589, 2006.

[8] P. D. Ariel, T. Hayat, and S. Asghar, "Homotopy perturbation method and axisymmetric flow over a stretching sheet," International Journal of Nonlinear Sciences and Numerical Simulation, vol. 7, no. 4, pp. 399-406, 2006.

[9] M. M. Mousa and A. Kaltayev, “Application of He's homotopy perturbation method for solving fractional Fokker-Planck equations," Zeitschrift fur Naturforschung, vol. 64, no. 12, pp. 788-794, 2009.

[10] A. Yildirim and S. A. Sezer, "Non-perturbative solution of the magnetohydrodynamic flow over a nonlinear stretching sheet by homotopy perturbation method-padé technique," Zeitschrift Fur Naturforschung, vol. 65, no. 12, pp. 1106-1110, 2010.

[11] A. M. Siddiqui, R. Mahmood, and Q. K. Ghori, "Homotopy perturbation method for thin film flow of a fourth grade fluid down a vertical cylinder," Physics Letters, Section A, vol. 352, no. 4-5, pp. 404-410, 2006.

[12] S. Das and P. K. Gupta, "An approximate analytical solution of the fractional diffusion equation with absorbent term and external force by homotopy perturbation method," Zeitschrift Fur Naturforschung, vol. 65, no. 3, pp. 182-190, 2010.

[13] R. Sakthivel, C. Chun, and J. Lee, "New travelling wave solutions of burgers equation with finite transport memory," Zeitschrift Fur Naturforschung, vol. 65, no. 8, pp. 633-640, 2010.

[14] M. Javidi and A. Golbabai, "A numerical solution for solving system of Fredholm integral equations by using homotopy perturbation method," Applied Mathematics and Computation, vol. 189, no. 2, pp. 1921-1928, 2007.

[15] M. M. Rashidi, D. D. Ganji, and S. Dinarvand, "Explicit analytical solutions of the generalized Burger and Burger-Fisher equations by homotopy perturbation method," Numerical Methods for Partial Differential Equations, vol. 25, no. 2, pp. 409-417, 2009.

[16] C. Chun, H. Jafari, and Y.-I. Kim, "Numerical method for the wave and nonlinear diffusion equations with the homotopy perturbation method," Computers \& Mathematics with Applications. An International Journal, vol. 57, no. 7, pp. 1226-1231, 2009.

[17] S. Dinarvand, "Viscous flow through slowly expanding or contracting porous walls with low seepage Reynolds number: a model for transport of biological fluids through vessels," Computer Methods in Biomechanics and Biomedical Engineering, vol. 14, no. 10, pp. 853-862, 2011.

[18] A. M. Siddiqui, T. Haroon, and S. Irum, "Torsional flow of third grade fluid using modified homotopy perturbation method," Computers $\mathcal{E}$ Mathematics with Applications, vol. 58, no. 11-12, pp. 2274-2285, 2009.

[19] M. S. H. Chowdhury and I. Hashim, "Solutions of Emden-Fowler equations by homotopyperturbation method," Nonlinear Analysis: Real World Applications, vol. 10, no. 1, pp. 104-115, 2009.

[20] Y. Khan, N. Faraz, A. Yildirim, and Q. Wu, "A series solution of the long porous slider," Tribology Transactions, vol. 54, no. 2, pp. 187-191, 2011.

[21] M. J. Stefan, "Versuch Über die scheinbare adhesion," Sitzungsberichten der Akademie der Wissenschaften in Wien, Mathematische Naturwissenschaften, vol. 69, pp. 713-721, 1874.

[22] O. Reynolds, "On the theory of lubrication," Transactions of the Royal Society, vol. 177, no. 1, pp. 157$234,1886$.

[23] F. R. Archibald, "Load capacity and time relations in squeeze films," Transactions of the ASME, Journal of Lubrication Technology, vol. 78, pp. 29-35, 1956. 
[24] R. J. Grimm, "Squeezing flows of newtonian liquid films an analysis including fluid inertia," Applied Scientific Research, vol. 32, no. 2, pp. 149-166, 1976.

[25] W. A. Wolfe, "Squeeze film pressures," Applied Scientific Research, vol. 14, no. 1, pp. 77-90, 1965.

[26] D. C. Kuzma, "Fluid inertia effects in squeeze films," Applied Scientific Research, vol. 18, no. 1, pp. 15-20, 1968.

[27] R. J. Grimm, "Squeezing flows of newtonian liquid films an analysis including fluid inertia," Applied Scientific Research, vol. 32, no. 2, pp. 149-166, 1976.

[28] J. A. Tichy and W. O. Winer, "Inertial considerations in parallel circular squeeze film bearings," Transactions of the ASME, Journal of Lubrication Technology, vol. 92, pp. 588-592, 1970.

[29] C. Y. Wang and L. T. Watson, "Squeezing of a viscous fluid between elliptic plates," Applied Scientific Research, vol. 35, no. 2-3, pp. 195-207, 1979.

[30] R. Usha and R. Sridharan, "Arbitrary squeezing of a viscous fluid between elliptic plates," Fluid Dynamics Research, vol. 18, no. 1, pp. 35-51, 1996.

[31] Y. Khan, Q. Wu, N. Faraz, A. Yildirim, and S. T. Mohyud-Din, "Three-dimensional flow arising in the long porous slider: an analytic solution," Zeitschrift fur Naturforschung, vol. 66, no. 8-9, pp. 507-511, 2011.

[32] M. H. Hamdan and R. M. Barron, "Analysis of the squeezing flow of dusty fluids," Applied Scientific Research, vol. 49, no. 4, pp. 345-354, 1992.

[33] N. Phan-Thien, "Squeezing flow of a viscoelastic solid," Journal of Non-newtonian Fluid Mechanics, vol. 95, no. 2-3, pp. 343-362, 2000.

[34] J. D. Jackson, "A study of squeezing flow," Applied Scientific Research, Section A, vol. 11, no. 1, pp. 148-152, 1963.

[35] S. Ishizawa, "The unsteady flow between two parallel discs with arbitary varying gap width," Bulletin of the Japan Society of Mechanical Engineers, vol. 9, no. 35, pp. 533-550, 1966.

[36] C. Y. Wang, "Squeezing of a fluid between two plates," American Society of Mechanical Engineers, vol. 43, no. 76, pp. 579-583, 1976.

[37] J.-H. He, "Homotopy perturbation technique," Computer Methods in Applied Mechanics and Engineering, vol. 178, no. 3-4, pp. 257-262, 1999.

[38] Z. Z. Ganji and D. D. Ganji, "Approximate solutions of thermal boundary-layer problems in a semiinfinite flat plate by using he's homotopy perturbation method," International Journal of Nonlinear Sciences and Numerical Simulation, vol. 9, no. 4, pp. 415-422, 2008.

[39] A. M. Siddiqui, M. A. Rana, S. Irum, R. Qamar, and T. Haroon, "Application of homotopy perturbation and numerical methods-to the unsteady squeezing mhd flow of a second grade fluid between circular plates," International Journal of Nonlinear Sciences and Numerical Simulation, vol. 10, no. 6, pp. 699-709, 2009.

[40] M. S. H. Chowdhury and I. Hashim, "Solutions of time-dependent Emden-Fowler type equations by homotopy-perturbation method," Physics Letters. A, vol. 368, no. 3-4, pp. 305-313, 2007.

[41] E. Hesameddini and H. Latifizadeh, "An Optimal Choice of Initial Solutions in the Homotopy Perturbation Method," International Journal of Nonlinear Sciences and Numerical, vol. 10, pp. 1389-1398, 2009.

[42] E. Hesameddini and H. Latifizadeh, "A new vision of the he's homotopy perturbation method," International Journal of Nonlinear Sciences and Numerical Simulation, vol. 10, no. 11-12, pp. 1415-1424, 2009. 


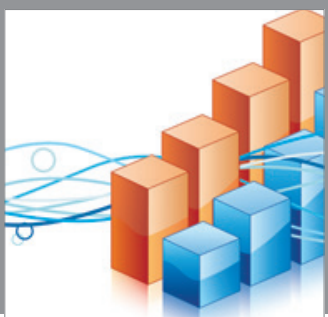

Advances in

Operations Research

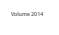

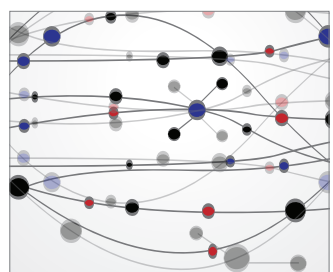

\section{The Scientific} World Journal
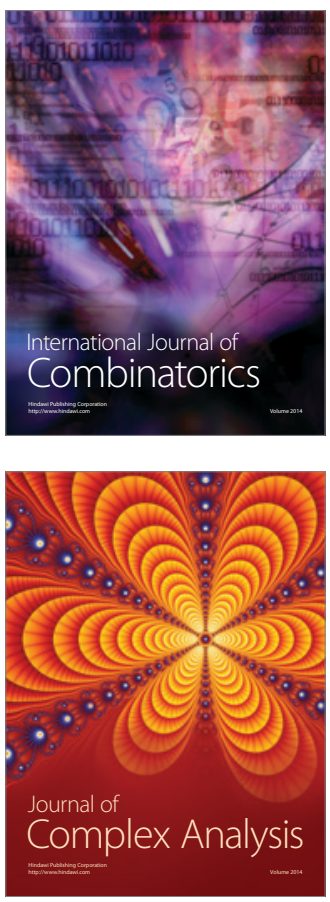

International Journal of

Mathematics and

Mathematical

Sciences
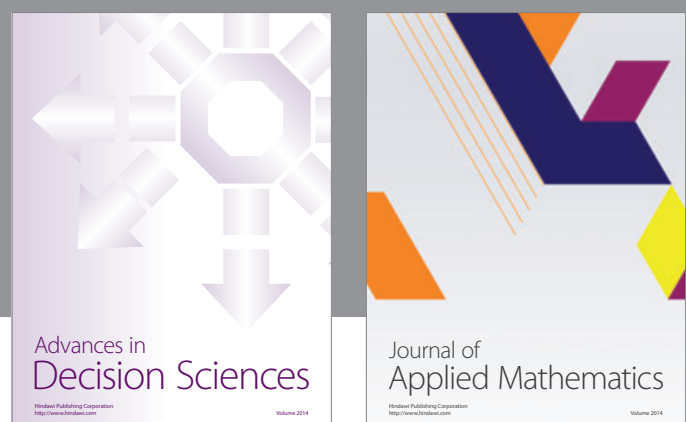

Journal of

Applied Mathematics
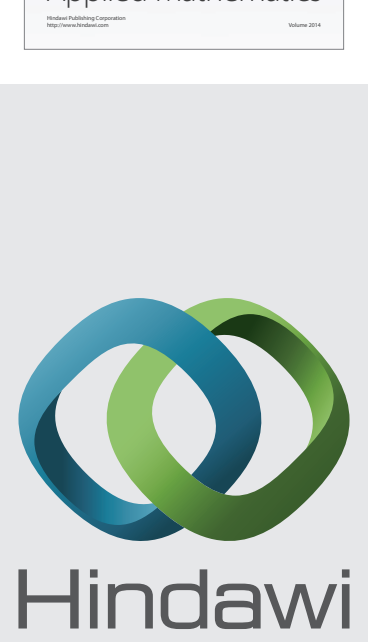

Submit your manuscripts at http://www.hindawi.com
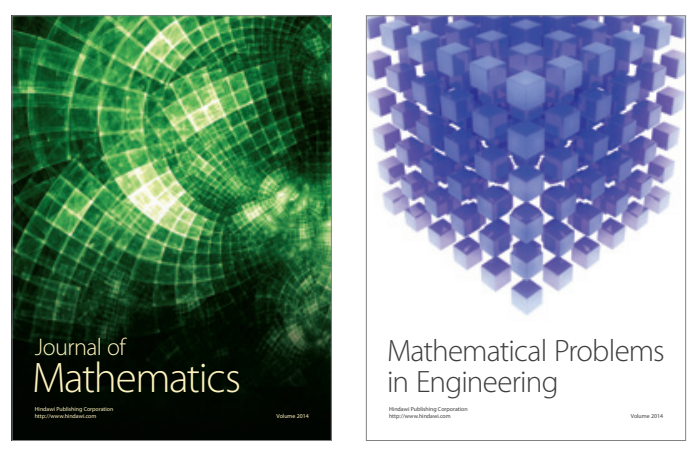

Mathematical Problems in Engineering
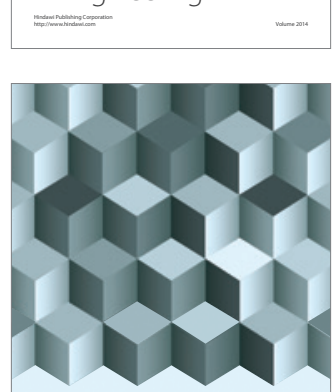

Journal of

Function Spaces
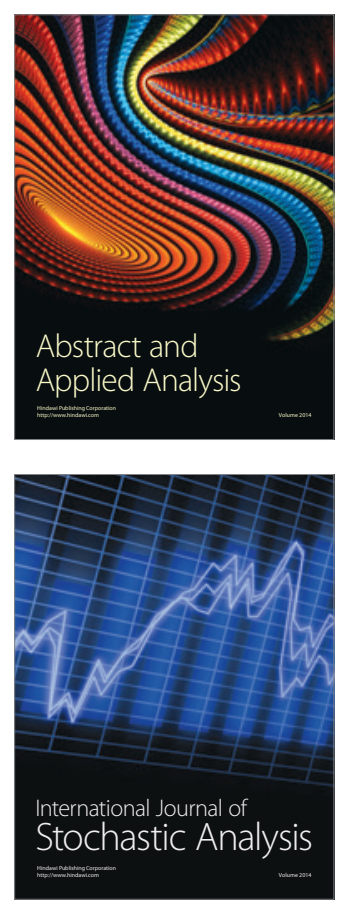

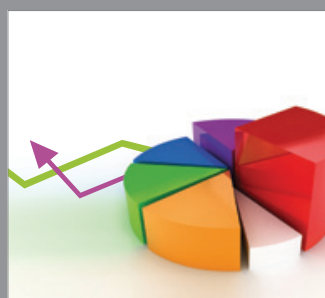

ournal of

Probability and Statistics

Promensencen
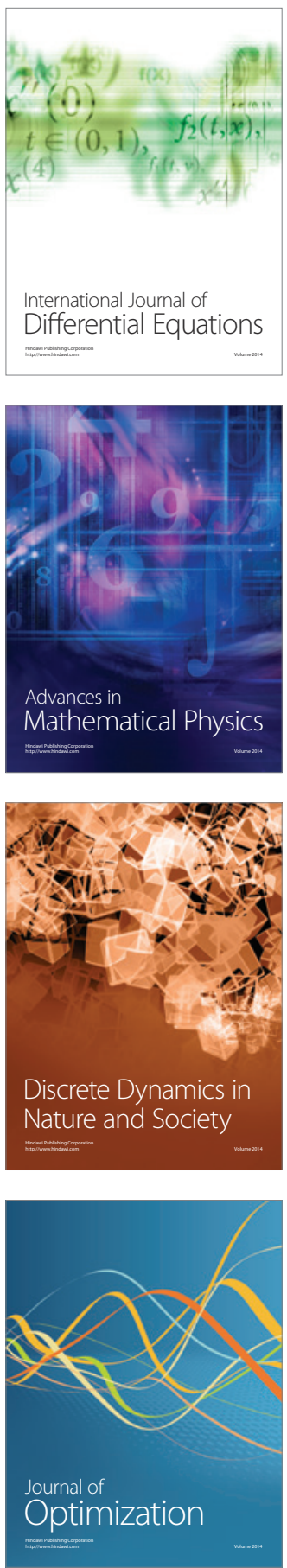